\title{
(S)PAR - die gebotene Transparenz im Studienwald
}

\section{Gert Printzen}

Dr. med., Mitglied des Zentralvorstandes der FMH, Departementsverantwortlicher Heilmittel

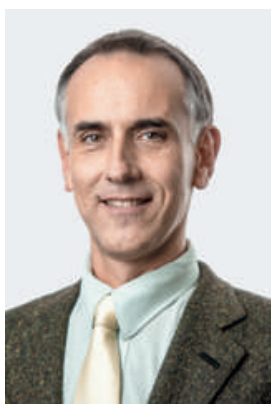

Dieses Editorial entstand als gemeinsames Anliegen von Seiten FMH und pharmaSuisse.
Bei der Abkürzung PAR handelt es sich um die «Publication of Assessment Reports of Marketing Authorization Applications", also um die Veröffentlichung der Anträge auf Marktzulassung und der jeweiligen Beurteilungsberichte der Zulassungsbehörde. Diese Veröffentlichungspflicht ist in der Schweiz seit 2014 im Öffentlichkeitsgesetz (BGÖ) verankert. Dies gilt auch für die öffentliche Verwaltung und damit auch für Swissmedic, was sich jedoch bis anhin nicht entsprechend niederschlug. So war es mit den bisher von Swissmedic zu einer Medikamentenzulassung in der Schweiz publizierten Informationen kaum möglich, herauszufinden, welche klinischen Versuche Grundlage dieser Zulassung bildeten. Auf die berechtigte Frage «Warum war das so?» wird in den anschliessenden Antworten immer wieder auf das sogenannte Firmengeheimnis verwiesen. Allerdings können gemäss einer Stellung-

Aufgrund des öffentlichen Interesses an der PARThematik fordern FMH und pharmaSuisse möglichst rasch eine ordentliche Vernehmlassung.

nahme ${ }^{1}$ des Instituts für Qualität und Wirtschaftlichkeit im Gesundheitswesen (IQWiG) ausgedehnte Geschäftsgeheimnis-Definitionen bei klinischen Studien die Transparenz gefährden.

1 Institut für Qualität und Wirtschaftlichkeit im Gesundheitswesen: Klinische Studien: Weite Geschäftsgeheimnis-Definition gefährdet Transparenz, online unter: https:// www.iqwig.de/de/presse/ pressemitteilungen/ pressemitteilungen/ klinische-studien-weitegeschaftsgeheimnis-definition-gefahrdet-transparenz.6599.html

2 «Nach Studienende müssen die Forscher der Kom mission einen Abschluss bericht vorlegen, der eine Zusammenfassung der Ergebnisse und Schlussfolgerungen der Studie enthält.» Vgl. deutsche Übersetzung der Deklaration von Helsinki, S. 6, abgerufen am 5. Februar 2016.
Im September 2015 führte Swissmedic das «Symposium on Transparency" zu dieser PAR-Thematik durch. Das Interesse der Zulassungsbehörde Swissmedic mag einerseits von den erkannten Lücken in diesem Bereich herrühren, andererseits von der 2014 angenommenen neuen EU-Verordnung über klinische Studien. Diese schreibt die systematische und verbindliche Veröffentlichung von klinischen Studien vor, die für die Zulassung der Medikamente als Entscheidungsgrundlage zur Verfügung stehen unabhängig vom Zulassungsentscheid. Direkt danach beauftragte die EU die europäische Arzneimittelzulassungsbehörde (European Medicines Agency, EMA) mit dem Aufbau einer Datenbank, in der künftig alle Interessenten umfassende Daten aus klinischen Studien einsehen können. Interessanterweise unterstrich das EU-Parlament, dass Daten aus klinischen Studien im
Allgemeinen keine Geschäftsgeheimnisse sind und nicht ohne weiteres von der Veröffentlichung in der Datenbank ausgenommen werden können. Darüber hinaus wäre eine Nichtveröffentlichung oder eine stark verzögerte Veröffentlichung von Studienergebnissen und -methoden auch nicht mit den Grundsätzen für Studien an Menschen vereinbar. ${ }^{2}$

Die Berufsverbände der Schweizer Ärzte und Apotheker, FMH und pharmaSuisse, stimmen überein in der Forderung, dass aufgrund des überwiegenden öffentlichen Interesses an der PAR-Thematik möglichst rasch eine ordentliche Vernehmlassung gerade auch unter Anhörung der involvierten Verbände erfolgen muss, um endlich eine praxisnahe und korrekte Umsetzung der gesetzlich vorgeschriebenen Bestimmungen zu gewährleisten.

Angesichts der anstehenden Entscheidungen ist es unabdingbar, dass die wissenschaftliche Unabhängigkeit gewahrt bleibt und keine Gewichtung und/oder Interpretation der Daten durch beispielsweise «scientific writers» vorgenommen wird. Gerade für die Diagnose, Indikationsstellung, Verschreibung sowie die fachlich beratende Abgabe auf individueller und evidenzbasierter Grundlage ist der Zugang zu ungefilterter und validierter Information eine unverzichtbare Voraussetzung. Diese unbefangene Herangehensweise gewährleistet eine kompetente und personalisierte Behandlung eines jeden Individuums. Hierfür sind Datenintegrität und wissenschaftliche Transparenz notwendig. Ob sich die Schweizer PAR-Variante in Anlehnung an

Gerade für die Diagnose, Indikationsstellung, Verschreibung sowie die fachlich beratende Abgabe ist der Zugang zu ungefilterter und validierter Information eine unverzichtbare Voraussetzung.

die Europäische Version (EPAR) als Swiss Public Assessment Reports (SPAR) etabliert, wird sich zeigen. Die Diskussionen am Swissmedic-Symposium lassen hoffen, dass SPAR nicht zur Spar-Übung verkommt. Die Berufsverbände FMH und pharmaSuisse verfolgen das Dossier mit grösster Aufmerksamkeit und konstruktivem Engagement. 